\title{
Ellis - van Creveld Syndrome, (Chondroectodermal Dysplasia Syndrome) in a Gurkha Family
}

\author{
Captain J C Nainby-Luxmoore
}

BA, MB, BS, RAMC

Senior House Officer in Paediatrics

British Military Hospital, Hong Kong, BFPO 1

SUMMARY: The first reported case of Ellis - van Creveld syndrome in a Gurkha child is described, and the implications of the syndrome in this ethnic group are briefly considered.

\section{Introduction}

This autosomal recessive syndrome was first described by Ellis and van Creveld in $1940^{1}$. They reported three children with the following congenital abnormalities: ectodermal dysplasia (affecting the hair, teeth and nails), polydactyly, chondrodysplasia, and congenital "morbus cordis". The largest single study of this syndrome was presented by McKuisk in $1964^{2}$ when he reported 52 cases in a highly inbred Amish population in the USA. It has, however, never been previously documented in a Nepalese family.

\section{Case Report}

A female child was delivered by a 24 year old Gurkha Corporal's wife, para 1 , gravida 2 . The mother's first child, a boy, had been born eighteen months previously by forceps delivery in Nepal, but had died at 8 months of age due to meningitis. During this second pregnancy she was admitted to hospital twice, once at 33 weeks (by dates) because of polyhydramnios, and then again at 35 weeks because of abdominal pain, possible antepartum hacmorrhage and premature labour.

Labour started spontaneously at $35+3$ weeks by dates ( 33 weeks by scan). The first stage took 7 hours 45 minutes and was latterly augmented by oxytocin; the second stage took 6 minutes. The baby's birth weight was $2.100 \mathrm{~kg}$, and she was given mucus extraction and facial oxygen. Apgar scores were 8 at 1 minute, 9 at 5 minutes and 9 at 10 minutes. She was then transferred to an incubator.

General clinical examination revealed multiple external abnormalities. The limbs were short and the chest was small (Fig. 1). Six fingers were present on each hand, and short simian palmar creases with incurving little fingers were also present bilaterally (Fig. 2). Clefts were noted bilaterally between the great and second toes, and all the nails were small and dysplastic (Fig. 3). Two incisor teeth were clearly present in both upper and lower jaws (Fig. 4). There was also an ejection systolic murmur which was loudest at the left sternal edge, but there were no signs of cardiac failure. The passage of a nasogastric tube excluded oesophageal atresia.

She received regular oral feeds, but sixteen hours after delivery she developed profound hypoglycacmia, thermoregulatory disturbance and cardiac failure. Despite all resuscitative efforts, she died six hours later.
Radiological examination after death confirme्d prematurity, and revealed a normal vault and spine, a normal ribs. There was, however, symmetriest shortening of both the proximal and distal bones of the limbs, which were otherwise normally modelled. Ulrçor sided polydactyly was also confirmed.

At postmortem examination the pericardium n⿳⺈⿴囗十心 normal, and the heart was of normal size. There was, however, a common atrioventricular orifice and a singte outlet from the heart. This truncus arteriosus supplied both the systemic and pulmonary circulations. The m偖 systemic veins and arteries appeared to have a nognid configuration.

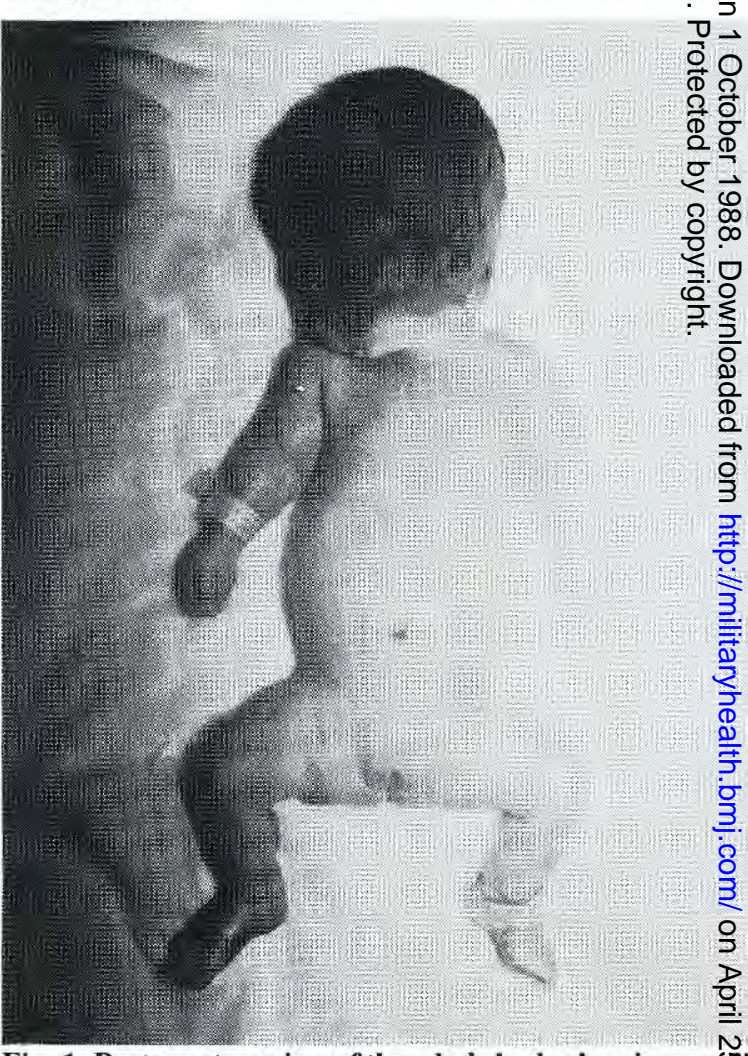

Fig. 1. Post mortem view of the whole body showing smsil chest and short extremities. 


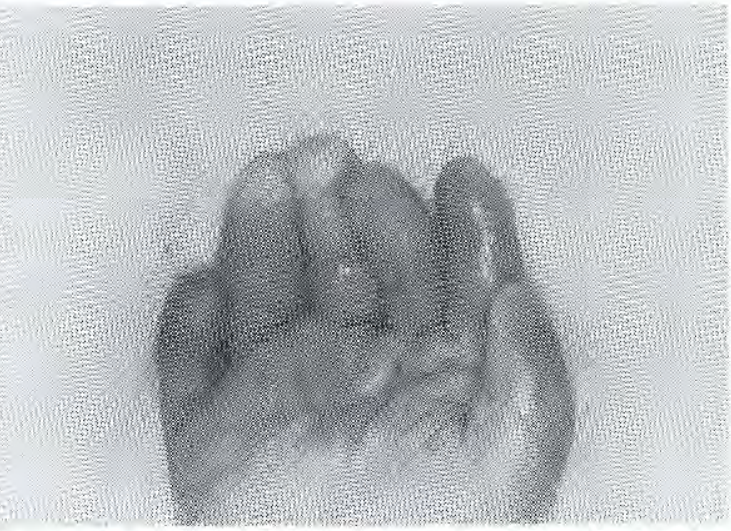

Fig. 2. Post mortem view of the right hand showing polydactyly.

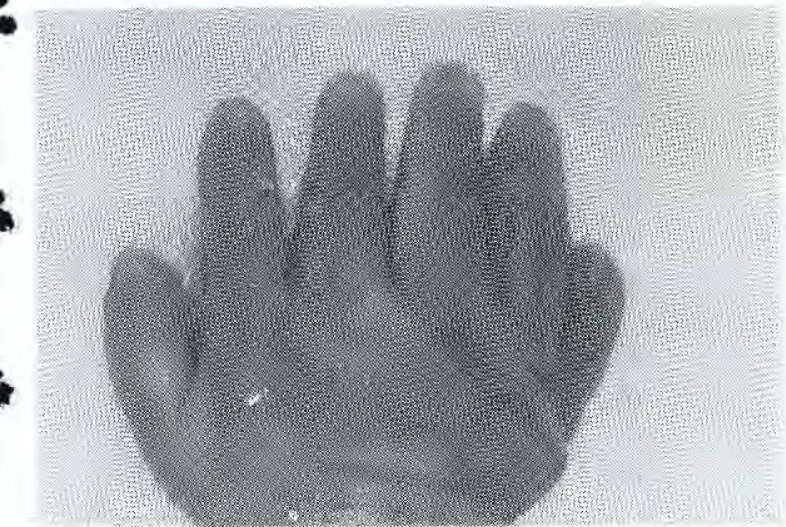

Fig. 3. Post mortem view of the dorsum of the right hand showing nail dysplasia and polydactyly.

\section{Discussion}

Ellis - van Creveld syndrome carries a mortality of about fifty per cent in early infancy because of the cardiac abnormalities. Most of those who survive are of normal intelligence and have an adult height of 43 to 60 inches. There are, however, frequent dental problems and also limitations in hand function.

As the syndrome has not previously been reported in a Nepalese family, its implications for these farming people have never been considered. While the short stature would be of little significance in a population of small people, the abnormalities of the hands and the possibility of mental retardation would not be socially acceptable. Nevertheless, Nepalese culture holds the ability to raise a large family in high esteem, despite high infant mortality, and male children receive markedly

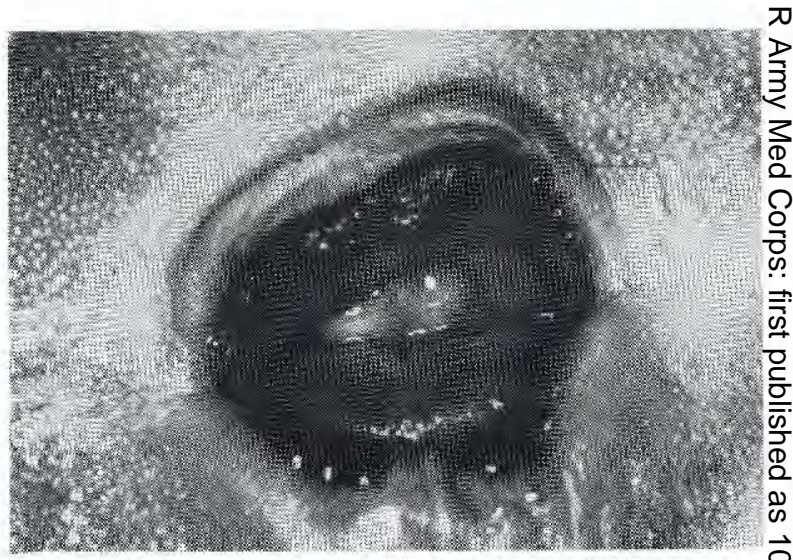

Fig. 4. Post mortem view of the mouth showing the upper two teeth.

preferential care. This family is, therefore currently of low status because both of their babies so far have died.

The autosomal recessive nature of the condition means that they are at high risk of having another affected child and would obviously benefit from antenatal diagnosis, as described in papers by Hobbis et $a l^{4}$, Mahoney et $a l^{5}$ and Bui et $a l^{6}$, using techniqu赵 such as real time ultrasound and fetoscopy. These are not, unfortunately, available in Nepal.

The question therefore arose as to whether the fami should be told of the $25 \%$ chance of subsequent babi being affected. In view of the social mores, it was fot that they would continue to try to have a family ung? they had at least one healthy boy. They were therefog told of the possibility of their having another affectei baby, but the numeric probability of such an event wo omitted.

\section{REFERENCES}

1. Ellis RWB and van Creveld S. A syndrome characterised by ectodermal dysplasia, polydactyly, chondro-dysplasia and congenital morbus cordis. Report of three cases. Arch Dis Child 1940; 15: 65-84.

2. ELIIS RWB and ANDREWS J D. Chondroectodermal dysplasia. J Bone Joint Surg 1962; 44B: 626-631.

3. McKuisk V A, et al. Dwarfism in the Amish. The Ellis - van Creveld syndrome. Bull Hopkins Hosp 1964; 115:306-336.

4. HoBbins J C. BRACKEN H B and MAHONEY H J. Diagnosis of fetal skeletal dysplasias with ultrasound. Am J Obstet Gynecol 1982; 142/3: 306-312

5. MAHONEY H J and HobBins J C. Prenatal diagnosis of chondroectodermal dysplasia (Ellis-van Creveld syndrome) 3 with fetoscopy and ultrasound. N Engl J Med 1977; 297(5): 0 258-260).

6. BUI T H $e l$ al. Prenatal diagnosis of chondrocctodermalo dysplasia with fetoscopy. Prenat Diagn. 1984; 4(2): 155-159. 